\title{
A INCLUSÃO DE PESSOAS COM DEFICIÊNCIA INTELECTUAL NO MERCADO DE TRABALHO, EM TRÊS LAGOAS/MS.
}

\author{
Silvana Cristina Fontanetti 1 \\ PintoPriscila Pereira Dias ${ }^{2}$ \\ Sandra Regina Ferreira Toresan Gonzales ${ }^{3}$
}

\section{RESUMO}

\begin{abstract}
O presente artigo retrata a experiência da inclusão de pessoas com deficiências intelectuais pela APAE na cidade de Três Lagoas, no estado do Mato Grosso do Sul, ao longo do período de 2011 a 2016. O crescimento do numero de incluídos nesse período foi de $12 \mathrm{em} 2011$, para 24 em 2016, com aumento de uma mulher em 2011 para 8 em 2016. As indústrias que mais contratam são as medias e pequenas, sobretudo as têxteis, de alimentos e de calçados infantis. Apesar do processo de treinamento e inclusão na empresa ser difícil e moroso, os resultados são satisfatórios, pois um deficiente foi promovido nesse período e vários solicitaram mudança de postos de trabalho e de empresa, não apenas por aumento de salários e sim buscando melhor qualidade do ambiente de trabalho $e$ consecutivo de vida.
\end{abstract}

PALAVRAS-CHAVE: deficiente intelectual - DI. Inclusão. Mercado do Trabalho. Qualidade de Vida.

\section{ABSTRACT}

This article portrays the experience of inclusion of people with intellectual disabilities by APAE in the city of Três Lagoas, in Mato Grosso do Sul, during the period from 2011 to 2016. The growth in the number of included in this period from 12 in 2011 to 24 in 2016, an increase of a woman in 2011 to 8 in 2016. Industries that hire more are the medium and small, especially textiles, food and children's shoes. Despite the training process and inclusion in the company to be difficult and time consuming, the results are satisfactory, because a disabled was promoted in this period and several requested change jobs and business, not only for wage increases but seeking better quality desktop and consecutive life.

\footnotetext{
${ }^{1}$ Pedagoga e Coordenadora do Programa 'Mercado de trabalho' da Associação de Pais e Amigos dos Excepcionais de Três Lagoas/MS (APAE/TL). E-mail: silvanafontanetti12@gmail.com

${ }^{2}$ Assistente Social da Associação de Pais e Amigos dos Excepcionais (APAE/TL) e do Tribunal de Justiça de Mato Grosso do Sul de três Lagoas. E-mail: priscila.morais@tjms.jus.br

${ }^{3}$ Psicóloga da Associação de Pais e Amigos dos Excepcionais de Três Lagoas/MS (APAE/TL). E-mail:

san_regin@hotmail.com
} 
KEYWORDS: intellectual disabled - DI. Inclusion. Labour market. Quality of life.

\section{INTRODUÇÃO}

Atualmente a inclusão de pessoas com deficiências tem sido um tema muito discutido, quer seja no âmbito social, escolar ou profissional. Apesar dos avanços obtidos através do movimento mundial pelos direitos humanos que vem repercutindo nas legislações de muitos países, contudo, este paradigma tem se mostrado de difícil desenvolvimento, pois requer um compromisso e cumplicidade da sociedade, na construção de um trabalho coletivo que garanta os direitos das pessoas com deficiência em todos os aspectos.

Dentre estes aspectos apresenta-se o profissional e sua inserção no mercado de trabalho. Tendo papel crucial o ensino, que amparado pelo artigo 59 da Lei de Diretrizes e Bases da Educação Nacional - LDB, que assegura que o sistema de ensino no Brasil, qualifique as pessoas com deficiência para o trabalho, visando à efetiva inclusão das pessoas com deficiência na vida em sociedade (BRASIL, 1996).

A cidade de Três Lagoas rapidamente se industrializou e passou a desenvolver forte atração populacional, bem acima da media do Mato Grosso do Sul, passando de uma população de 79.059, em 2000, para 113.619 habitantes, em 2015, sendo a terceira cidade mais populosa do Estado (MATO GROSSO DO SUL, 2015). E segundo a Federação das Indústrias do Estado do Rio de Janeiro (FIRJAN), o Índice de Desenvolvimento Municipal (IFDM), apontou em 2013, Três Lagoas como o segundo Município do Brasil e o primeiro do Estado de Mato Grosso do Sul com o maior índice de geração de emprego e renda, ao alcançar a pontuação de 0,8955 .

Esse grande crescimento populacional e de postos de trabalho, oportunizou, sobretudo amparado pela lei federal 8.213 de 24 de julho de 1991, que dispõe sobre a contratação de deficientes nas empresas, a abertura potencial de postos de trabalho para pessoas com deficiência. Contudo o desconhecimento dos potenciais de trabalho das pessoas com deficiência intelectual, bem como a sua desqualificação os deixava impossibilitados de concorrer a estas vagas. A APAE de Três Lagoas, através da sua equipe de inclusão de mercado de trabalho, inicia em 2003, programa com ações educativas de conscientização nas empresas, treinamento das pessoas com deficiência intelectual e acompanhamento dos inseridos nas empresas do município de Três Lagoas.

Como este trabalho cresceu, amparado pela parceria com as empresas do município e vem apresentando bons resultados, o presente trabalho tem como objetivo geral, avaliar as inclusões realizadas pela APAE Três Lagoas, nas empresas do município de Três Lagoas, no período de 2011 a 2016. 


\section{DESENVOLVIMENTO}

\subsection{Revisão Bibliográfica}

Segundo o Manual de Diagnóstico e Estatística de Distúrbios Mentais (1994) e os decretos $n^{\text {o }} 3.298 / 99$ e $n^{\text {o }} 5.296 / 04$ o deficiente intelectual é caracterizado por um funcionamento intelectual significativamente inferior à média, acompanhado de limitações significativas no funcionamento adaptativo, em pelo menos duas das seguintes áreas de habilidades: comunicação, autocuidados, vida doméstica, habilidades sociais/interpessoais, uso de recursos comunitários, autossuficiência, habilidades acadêmicas, trabalho, lazer, saúde e segurança.

A Constituição Federal de 1988 propõem avanços significativos para a educação escolar de pessoas com deficiência ao trazer como um dos seus objetivos fundamentais a promoção do bem de todos, sem preconceitos de origem, raça, sexo, cor, idade e quaisquer outras formas de discriminação (art. $3^{\circ}$, inciso IV). Ela garante ainda do direito à igualdade (art. $5^{\circ}$ ) e trata no artigo 205, o direito de todos a educação que visa o desenvolvimento pleno das pessoas, do seu preparo para cidadania e sua formação profissional para o trabalho.

Com a implementação da lei federal 8.213 de 1991, abre-se grande oportunidade de inclusão as pessoas com deficiência para o mercado de trabalho, sobre tudo em seu artigo 93, que estabelece cotas para as empresas. A empresa com 100 ou mais funcionários está obrigada a preencher de dois a cinco por cento dos seus cargos com beneficiários reabilitados, ou pessoas portadoras de deficiência, na seguinte proporção:

$$
\begin{aligned}
& \text { - até } 200 \text { funcionários.................. 2\% } \\
& \text { - de } 201 \text { a } 500 \text { funcionários............ 3\% } \\
& \text { - de } 501 \text { a } 1000 \text { funcionários......... 4\% } \\
& \text { - de } 1001 \text { em diante....................... 5\%. }
\end{aligned}
$$

Essa legislação é complementada com a exigência de que os sistemas de ensino devam assegurar a qualificação para o trabalho, visando à efetiva inclusão das pessoas com deficiência na vida em sociedade (Lei de Diretrizes e Bases da Educação Nacional - LDB) (BRASIL, 1996, art.59).

As empresas precisam contratar trabalhadores. No entanto, é evidente a problemática existente, qual seja a de encontrar muitos deficientes desqualificados para as vagas existentes no mercado de trabalho.

As empresas precisam de uma estrutura para recebê-los e estes, de capacitação e interesse em desenvolver seu lado intelectual para ocuparem tais vagas. Cabe aos órgãos governamentais, capacitá-los e às empresas, disponibilizar espaço físico para que todos 
consigam alcançar o interesse comum: formar e integrar cidadãos dignos do trabalho diário (MACHADO, 2012).

\subsection{Procedimentos Metodológicos}

Para a elaboração da pesquisa, inicialmente serão levantados todas as pessoas com deficiência intelectual que foram treinados pela Associação de Pais e Amigos dos Excepcionais - APAE de Três Lagoas, no período de 2011 a 2016. Para assegurar que estes fiquem anônimos, cada aluno receberá um número e será tratado ao longo da pesquisa por ele.

Posteriormente, será tabulado por sexo, faixa etária, data de admissão, nome da empresa, tempo de treinamento, data de demissão, causa da demissão, para cada ano desse período.

Em seguida, será levantado como é, e qual o tempo médio de realização do treinamento das pessoas com deficiência intelectual, pela APAE Três Lagoas, bem como, é efetuado o estudo do potencial posto de trabalho, da adequação do treinamento a vaga, e de todo processo de inclusão, desde a sensibilização do setor onde será inserido, até o seu acompanhamento.

Os dados serão ordenados e tabulados por ano, e a pesquisa partirá do particular para o geral, isto é, partirá do ano de 2011, seguindo por anos até 2016 e findará com a analise do período. Para facilitar a analise, os dados serão trabalhados em Excel.

Portanto, para cada ano será construído tabelas, apresentando a realidade da inclusão de cada ano, número de incluídos no ano anterior, no ano, por sexo, idade, classe de ocupação, tempo de treinamento, tempo de serviço na empresa, se já foi reintroduzido, quando ocorreu e motivo da saída.

Para finalizar será efetuada a analise do período de 2011 a 2016.

\subsection{Resultados}

Em 2011 a APAE de Três Lagoas possuía inserido no mercado de trabalho da cidade de Três Lagoas 13 deficientes intelectuais - DI, em 8 empresas, oscilando entre as classes de idade de 20 a 45 anos, sendo apenas uma mulher, que atuava na empresa Cargill Produtos Agrícolas. A empresa que mais empregava deficientes intelectuais, neste ano foi a Pepsico Mabel, com três inseridos, Tabela 1.

A ocupação funcional na qual maior número de DIs foram inseridos no mercado de trabalho de Três lagoas, em 2011 foi de auxiliar de produção, com cinco homens. 
$\mathrm{O}$ fato mais marcante neste ano e que demonstra o bom treinamento da APAE Três Lagoas e a possibilidade de atingir eficiência de função repetitiva, deu-se na empresa Pepsico Mabel, com a promoção de um DI, contratado pela empresa desde 2007, que passou, neste ano, de repositor para supervisor de repositor.

Tabela 1. DIs Inseridos e Acompanhados pela APAE de Três Lagoas/MS, em 2011.

\begin{tabular}{|r|c|c|c|c|l|l|}
\hline $\mathbf{N}^{\mathbf{0}}$ & Sexo & Def. & Idade & Admissão & \multicolumn{1}{|c|}{ Função } & \multicolumn{1}{|c|}{ Empresa } \\
\hline $\mathbf{1}$ & M & DI & 36 & 2003 & Empacotador & Proença Supermercados \\
\hline $\mathbf{2}$ & M & DI & 34 & 2007 & Supervisor de Repositor & Pepsico Mabel \\
\hline $\mathbf{3}$ & F & DI & 26 & 2009 & Auxiliar de Serviços Gerais & Cargill Produtos Agrícolas \\
\hline $\mathbf{4}$ & M & DI & 27 & 2009 & Auxiliar de Produção & Pepsico Mabel \\
\hline $\mathbf{5}$ & M & DI & 33 & 2011 & Empacotador & Proença Supermercados \\
\hline $\mathbf{6}$ & M & DI & 38 & 2009 & Auxiliar de Almoxarifado & Cargill Produtos Agrícolas \\
\hline $\mathbf{7}$ & M & DI & 38 & 2005 & Empacotador & Supermercado Nova Estrela \\
\hline $\mathbf{8}$ & M & DI & 23 & 2007 & Auxiliar de Serviços Gerais & Avanti Ind. e Com. Imp. e Exp. \\
\hline $\mathbf{9}$ & M & DI & 29 & 2007 & Auxiliar de Produção & Pepsico Mabel \\
\hline $\mathbf{1 0}$ & M & DI & 44 & 2011 & Auxiliar de Produção & Klin Produtos Infantis \\
\hline $\mathbf{1 1}$ & M & DI & 25 & 2006 & Repositor & Posto Parati \\
\hline $\mathbf{1 2}$ & M & DI & 38 & 2011 & Auxiliar de Produção & Klin Produtos Infantis \\
\hline $\mathbf{1 3}$ & M & DI & 26 & 2010 & Aux. de Produção & Fatex Indústria e Comercio \\
\hline
\end{tabular}

Em 2012 a APAE possuía 17 deficientes intelectuais inseridos em 8 empresas trêslagoenses, sendo que apenas 4 novos alunos foram inseridos e dois solicitaram mudança de empresa, por alegarem conhecer novo posto de trabalho e novos colegas. Sendo que apenas um nesse ano foi reintroduzido, saindo da empresa Proença Supermercados, como empacotador e foi para a Pepsico Mabel, como auxiliar de produção. O outro que solicitou mudança de empresa e de posto de trabalho voltou para a APAE para fazer novos treinamentos para possibilitar sua entrada no ano seguinte, na empresa Klin Produtos Infantis. Empresa esta, que em 2012 foi a que mais empregou DIs, totalizando cinco inseridos, Tabela 2.

Outro fato importante é que neste ano, o numero de mulheres inseridas subiu de uma para quatro, sendo duas na empresa Cargill Produtos Agrícolas e duas na Klin Produtos Infantis. 
Tabela 2. DIs Inseridos e Acompanhados pela APAE de Três Lagoas/MS, em 2012.

\begin{tabular}{|l|l|l|l|l|l|c|l|l|}
\hline $\mathbf{N}^{\mathbf{o}}$ & Sexo & Def. & Idade & Admissão & Desligado & $\begin{array}{c}\text { Motivo da } \\
\text { Saída }\end{array}$ & \multicolumn{1}{|c|}{ Função } & \multicolumn{1}{|c|}{ Empresa } \\
\hline $\mathbf{0 1}$ & M & DI & 37 & 2003 & - & - & Empacotador & $\begin{array}{l}\text { Proença } \\
\text { Supermercado }\end{array}$ \\
\hline $\mathbf{0 2}$ & M & DI & 30 & 2007 & - & - & $\begin{array}{l}\text { Supervisor } \\
\text { Repositor }\end{array}$ & Pepsico Mabel \\
\hline $\mathbf{0 3}$ & F & DI & 27 & 2009 & - & - & $\begin{array}{l}\text { Aux. de Serviços } \\
\text { Gerais }\end{array}$ & Cargill \\
\hline $\mathbf{0 4}$ & F & DI & 27 & 2011 & - & - & Aux. de Produição & Klin \\
\hline $\mathbf{0 5}$ & M & DI & 28 & 2009 & 2012 & $\begin{array}{c}\text { Conhecer } \\
\text { P.Trab. }\end{array}$ & Aux. de Produção & Pepsico Mabel \\
\hline $\mathbf{0 6}$ & F & DI & 19 & 2012 & - & - & Aux. de Copeira & Cargill \\
\hline $\mathbf{0 7}$ & M & DI & 34 & 2009 & 2012 & $\begin{array}{c}\text { Conhecer } \\
\text { P.Trab. }\end{array}$ & Empacotador & $\begin{array}{l}\text { Proença } \\
\text { Supermercado }\end{array}$ \\
\hline $\mathbf{- 1}$ & - & - & - & 2012 & - & - & Aux. de Produção & Pepsico Mabel \\
\hline $\mathbf{0 8}$ & M & DI & 39 & 2009 & - & - & Aux. de Almoxarifado & Cargill \\
\hline $\mathbf{0 9}$ & F & DI & 22 & 2012 & - & - & Aux. de Produção & Klin \\
\hline $\mathbf{1 0}$ & M & DI & 39 & 2005 & - & - & Empacotador & Sup. Nova Estrela I \\
\hline $\mathbf{1 1}$ & M & DI & 24 & 2007 & - & - & $\begin{array}{l}\text { Aux. de Serviços } \\
\text { Gerais }\end{array}$ & Avanti \\
\hline $\mathbf{1 2}$ & M & DI & 30 & 2007 & - & - & Aux. de Produção & Pepsico Mabel \\
\hline $\mathbf{1 3}$ & M & DI & 45 & 2011 & - & - & Aux. de Produção & Klin \\
\hline $\mathbf{1 4}$ & M & DI & 26 & 2006 & - & - & Repositor & Posto Parati \\
\hline $\mathbf{1 5}$ & M & DI & 39 & 2011 & - & - & Aux. de Produção & Klin \\
\hline $\mathbf{1 6}$ & M & DI & 36 & 2012 & - & - & Aux. de Produção & Klin \\
\hline $\mathbf{1 7}$ & M & DI & 27 & 2010 & - & - & Aux. de Produção & Fatex \\
\hline
\end{tabular}

Em 2013 o numero de inseridos subiu de 17 para 25, com a contratação de duas mulheres e seis homens, permanecendo ainda a empresa Klin Produtos Infantis, como a maior parceira da APAE, todos atuando como auxiliares de produção, Tabela 3. E o numero de parceiros passou de 8 para 12 empresas, mostrando a confiança no trabalho da APAE Três Lagoas, no desempenho dos inseridos, visto que os motivos que estes são demitidos, refere-se a solicitação por parte dos DIs de troca de empresa e de postos de trabalho e não por insatisfação da empresa.

Neste ano dois DIs foram afastados do trabalho por licença medica, por desenvolverem doenças graves que os impossibilitam de trabalhar e que estão solicitando aposentadoria, Tabela 3. 
Tabela 3.DIs Inseridos e Acompanhados pela APAE de Três Lagoas/MS, em 2013.

\begin{tabular}{|c|c|c|c|c|c|c|c|c|}
\hline $\mathbf{N}^{\mathbf{o}}$ & Sexo & Def. & Idade & Admissão & Desligado & $\begin{array}{c}\text { Motivo da } \\
\text { Saída } \\
\end{array}$ & FUNÇÃO & EMPRESA \\
\hline 01 & $\mathrm{M}$ & DI & 38 & 2003 & 2013 & $\begin{array}{l}\text { Conhecer } \\
\text { P.Trab. }\end{array}$ & Empacotador & $\begin{array}{l}\text { Proença } \\
\text { Supermercado }\end{array}$ \\
\hline - & - & - & & 2013 & - & - & Empacotador & Sup. Nova Estrela \\
\hline 02 & $\mathrm{M}$ & DI & 33 & 2013 & - & - & Auxiliar de Limpeza & Cargill \\
\hline $\mathbf{0 3}$ & $\mathrm{M}$ & DI & 31 & 2007 & - & - & $\begin{array}{ll}\text { Supervisor } & \text { de } \\
\text { Repositor } & \\
\end{array}$ & Pepsico Mabel \\
\hline 04 & $\mathrm{~F}$ & DI & 28 & 2009 & - & - & $\begin{array}{lll}\text { Aux. } & \text { de } & \text { Serviços } \\
\text { Gerais } & & \\
\end{array}$ & Cargill \\
\hline 05 & $\mathrm{~F}$ & DI & 39 & 2013 & - & - & $\begin{array}{l}\text { Repositora/Aux. de } \\
\text { Caixa }\end{array}$ & Pepsico Mabel \\
\hline 06 & $\mathrm{~F}$ & DI & 28 & 2013 & - & - & $\begin{array}{lll}\begin{array}{l}\text { Atendente de } \\
\text { Gerais }\end{array} & \text { Serv. } \\
\end{array}$ & Enterhome \\
\hline 07 & $\mathrm{~F}$ & DI & 28 & 2011 & - & - & Auxiliar de Produção & Klin \\
\hline 08 & $\mathrm{~F}$ & DI & 20 & 2012 & - & - & Aux. de Copeira & Cargill \\
\hline 09 & $\mathrm{M}$ & DI & 29 & 2013 & - & - & Aux. de Produção & Klin \\
\hline 10 & $\mathrm{M}$ & DI & 35 & 2012 & - & - & Aux. de Produção & Pepsico Mabel \\
\hline 11 & $\mathrm{M}$ & DI & 40 & 2009 & - & - & Aux. de Almoxarifado & Cargill \\
\hline 12 & $\mathrm{~F}$ & DI & 23 & 2012 & - & - & Aux. de Produção & Klin \\
\hline 13 & $\mathrm{M}$ & DI & 27 & 2013 & - & - & Aux. de Produção & Klin \\
\hline 14 & $\mathrm{M}$ & DI & 40 & 2005 & - & - & Empacotador & Sup. Nova Estrela I \\
\hline 15 & $\mathrm{M}$ & DI & 25 & 2007 & - & - & $\begin{array}{lll}\text { Aux. } & \text { de } & \text { Serviços } \\
\text { Gerais } & & \\
\end{array}$ & Avanti \\
\hline 16 & $\mathrm{M}$ & DI & 31 & 2007 & - & - & Aux. de Produção & Pepsico Mabel \\
\hline 17 & $\mathrm{M}$ & DI & 35 & 2013 & - & - & Aux. de Caixa & $\begin{array}{l}\text { Sup. Nova Estrela } \\
\text { III }\end{array}$ \\
\hline 18 & $\mathrm{M}$ & DI & 46 & 2011 & - & - & Aux. de Produção & Klin \\
\hline 19 & $\mathrm{M}$ & DI & 28 & 2007 & - & - & $\begin{array}{lll}\text { Aux. } & \text { de } & \text { Serviços } \\
\text { Gerais } & \end{array}$ & $\begin{array}{l}\text { Transporte } \\
\text { Luiz }\end{array}$ \\
\hline 20 & $\mathrm{M}$ & DI & 27 & 2006 & - & - & Repositor & Posto Parati \\
\hline 21 & $\mathrm{M}$ & DI & 40 & 2011 & - & - & Aux. de Produção & Klin \\
\hline 22 & M & DI & 37 & 2012 & 2013 & Saúde & Aux. de Produção & Klin \\
\hline 23 & $\mathrm{M}$ & DI & 28 & 2010 & - & - & Aux. de Produção & Fatex \\
\hline 24 & $\mathrm{~F}$ & DI & 40 & 2013 & - & - & Auxiliar de Recepção & Clinica CERII \\
\hline 25 & $\mathrm{M}$ & DI & 18 & 2013 & - & - & Aux. de Produção & $\begin{array}{l}\text { Alimentos Sta. } \\
\text { Cruz }\end{array}$ \\
\hline
\end{tabular}

Em 2014 o numero de parceiros caiu para 11 empresas, a Klin, Produtos Infantis permanece como a maior parceira, com seis inseridos e em segundo lugar a Cargill Produtos Agrícolas, com cinco. Vale ressaltar que o plano de cargo e salários, e o plano de saúde da Cargill Produtos Agrícolas são os melhores entre as empresas, atraindo maior interesse entre os DIs, mostrando que não são ingênuos, Tabela 4.

Neste ano ocorreram as duas primeiras demissões dos inseridos pela APAE Três Lagoas, ambas por motivo de comportamento inadequado, sendo uma na empresa FATEX Indústria e Comercio e outra na Cargill Produtos Agrícolas. 
Tabela 4. DIs Inseridos e Acompanhados pela APAE de Três Lagoas/MS, em 2014.

\begin{tabular}{|c|c|c|c|c|c|c|c|c|}
\hline $\mathbf{N}^{\circ}$ & \begin{tabular}{|l|} 
Sexo \\
\end{tabular} & Def. & Idade & $\begin{array}{l}\text { Admis- } \\
\text { são }\end{array}$ & Desligado & $\begin{array}{l}\text { Motivo da } \\
\text { Saída }\end{array}$ & Função & EMPRESA \\
\hline 01 & $\mathrm{M}$ & DI & 39 & 2013 & 2014 & $\begin{array}{l}\text { Conhecer } \\
\text { P.Trab. }\end{array}$ & Empacotador & Sup. Nova Estrela \\
\hline 02 & M & DI & 34 & 2013 & 2014 & Comportamento & Auxiliar de Limpeza & Cargill \\
\hline- & - & - & - & 2014 & - & - & $\begin{array}{l}\text { Aux. De Serviços } \\
\text { Gerais }\end{array}$ & Avanti \\
\hline 03 & $\mathrm{M}$ & DI & 32 & 2007 & - & - & $\begin{array}{l}\text { Supervisor de } \\
\text { Repositor }\end{array}$ & Pepsico Mabel \\
\hline 04 & $\mathrm{~F}$ & DI & 29 & 2009 & - & - & $\begin{array}{l}\text { Aux. de Serviços } \\
\text { Gerais }\end{array}$ & Cargill \\
\hline 05 & $\mathrm{~F}$ & DI & 39 & 2013 & - & - & $\begin{array}{l}\text { Repositor/Aux. de } \\
\text { Caixa }\end{array}$ & Pepsico Mabel \\
\hline 06 & $\mathrm{~F}$ & DI & 29 & 2013 & - & - & $\begin{array}{l}\text { Atendente de Serv. } \\
\text { Gerais }\end{array}$ & Enterhome \\
\hline 07 & $\mathrm{~F}$ & DI & 29 & 2011 & - & - & $\begin{array}{l}\text { Auxiliar de } \\
\text { Produção }\end{array}$ & Klin \\
\hline 08 & $\mathrm{~F}$ & DI & 21 & 2012 & - & - & Aux. de Copeira & Cargill \\
\hline 09 & M & DI & 30 & 2013 & - & - & Aux. de Produção & Klin \\
\hline 10 & M & DI & 36 & 2012 & - & - & Aux. de Produção & Pepsico Mabel \\
\hline 11 & M & DI & 41 & 2009 & - & - & $\begin{array}{l}\text { Aux. de } \\
\text { Almoxarifado }\end{array}$ & Cargill \\
\hline 12 & $\mathrm{~F}$ & DI & 24 & 2012 & - & - & Aux. de Produção & Klin \\
\hline 13 & M & DI & 24 & 2014 & - & - & Aux. de Limpeza & Cargill \\
\hline 14 & M & DI & 28 & 2013 & - & - & Aux. de Produção & Klin \\
\hline 15 & $\mathrm{M}$ & DI & 41 & 2005 & - & - & Empacotador & $\begin{array}{l}\text { Sup. Nova Estrela } \\
\text { I }\end{array}$ \\
\hline 16 & M & DI & 26 & 2007 & - & - & $\begin{array}{l}\text { Aux. de Serviços } \\
\text { Gerais }\end{array}$ & Avanti \\
\hline 17 & M & DI & 32 & 2007 & - & - & Aux. de Produção & Pepsico Mabel \\
\hline 18 & M & DI & 36 & 2013 & - & - & Aux. de Caixa & Sup. Nova Estrela \\
\hline 19 & M & DI & 47 & 2011 & - & - & Aux. de Produção & Klin \\
\hline 20 & M & DI & 29 & 2007 & - & - & $\begin{array}{l}\text { Aux. de Serviços } \\
\text { Gerais }\end{array}$ & $\begin{array}{l}\text { Transporte São } \\
\text { Luiz }\end{array}$ \\
\hline 21 & $\mathrm{M}$ & DI & 28 & 2006 & - & - & Repositor & Posto Parati \\
\hline 22 & $\mathrm{M}$ & DI & 41 & 2011 & - & - & Aux. de Produção & Klin \\
\hline 23 & M & DI & 29 & 2010 & 2014 & Comportamento & Aux. de Produção & Fatex \\
\hline 24 & $\mathrm{~F}$ & DI & 41 & 2013 & - & - & $\begin{array}{l}\text { Auxiliar de } \\
\text { Recepção }\end{array}$ & Clinica CERII \\
\hline 25 & $\mathrm{M}$ & DI & 19 & 2013 & - & - & Aux. de Produção & $\begin{array}{l}\text { Alimentos Sta. } \\
\text { Cruz }\end{array}$ \\
\hline
\end{tabular}

Em 2015, mesmo com a grande crise econômica que assola o país o numero de inseridos no mercado de trabalho, ainda assim, teve resultado positivo de 22 para 23 , bem como o numero de parceiros, passando de 11 para 12, Tabela 5.

A função de auxiliar de produção manteve-se em todos os anos analisados como a função de maior ocupação. O DI empregado na empresa Pesico Mabel, inserido nessa empresa e promovido em 2011, para supervisor de repositor, ainda manteve-se nesse cargo até 2015, mostrando o seu bom desempenho e que são capazes de superar, com esforço próprio e ajuda especializada, serem inseridos com sucesso e admirados por seu desempenho pela empresa. 
Tabela 5. DIs Inseridos e Acompanhados pela APAE de Três Lagoas/MS, em 2015.

\begin{tabular}{|c|c|c|c|c|c|c|c|c|}
\hline $\mathbf{N}^{\mathbf{o}}$ & Sexo & Def. & Idade & $\begin{array}{c}\text { Admis- } \\
\text { são }\end{array}$ & Desligado & $\begin{array}{l}\text { Motivo da } \\
\text { Saída }\end{array}$ & Função & Empresa \\
\hline 01 & $M$ & DI & 40 & 2015 & - & - & Aux. de Produção & Adar \\
\hline 02 & $\mathrm{M}$ & DI & 25 & 2015 & - & - & Aux. de Produção & Emplal \\
\hline $\mathbf{0 3}$ & $\mathrm{M}$ & DI & 35 & 2014 & - & - & $\begin{array}{l}\text { Aux. de Serviços } \\
\text { Gerais }\end{array}$ & Avanti \\
\hline 04 & $\mathrm{M}$ & DI & 33 & 2007 & - & - & $\begin{array}{l}\text { Supervisor de } \\
\text { Repositor }\end{array}$ & Pepsico Mabel \\
\hline 05 & $\mathrm{~F}$ & DI & 30 & 2009 & - & - & $\begin{array}{l}\text { Aux. de Serviços } \\
\text { Gerais }\end{array}$ & Cargill \\
\hline 06 & $\mathrm{~F}$ & DI & 41 & 2013 & - & - & $\begin{array}{l}\text { Repositora/Aux. de } \\
\text { Caixa }\end{array}$ & Pepsico Mabel \\
\hline 07 & $\mathrm{~F}$ & DI & 30 & 2013 & - & - & $\begin{array}{l}\text { Atendente de Serv. } \\
\text { Gerais }\end{array}$ & Enterhome \\
\hline 08 & $\mathrm{~F}$ & DI & 29 & 2012 & 2015 & Comportamento & Aux. de Limpeza & Cargill \\
\hline 09 & $\mathrm{~F}$ & DI & 30 & 2011 & 2015 & Saúde & Aux. de produção & Klin \\
\hline 10 & $\mathrm{~F}$ & DI & 22 & 2012 & - & - & Aux. de Copeira & Cargill \\
\hline 11 & M & DI & 31 & 2013 & - & - & Aux. de Produção & Klin \\
\hline 12 & $\mathrm{M}$ & DI & 37 & 2012 & 2015 & Saúde & Aux. de Produção & Pepsico Mabel \\
\hline 13 & $\mathrm{~F}$ & DI & 23 & 2015 & - & - & Aux. de Produção & Emplal \\
\hline 14 & $\mathrm{M}$ & DI & 42 & 2009 & - & - & $\begin{array}{l}\text { Aux. de } \\
\text { Almoxarifado }\end{array}$ & Cargill \\
\hline 15 & $\mathrm{~F}$ & DI & 25 & 2012 & - & - & Aux. de Produção & Klin \\
\hline 16 & $\mathrm{M}$ & DI & 25 & 2014 & - & - & Aux. de Limpeza & Cargill \\
\hline 17 & $M$ & DI & 29 & 2013 & 2015 & Saúde & Aux. de Produção & Klin \\
\hline 18 & M & DI & 42 & 2005 & - & - & Empacotador & Sup. Nova Estrela \\
\hline 19 & M & DI & 27 & 2007 & - & - & $\begin{array}{l}\text { Aux. de Serviços } \\
\text { Gerais }\end{array}$ & Avanti \\
\hline 20 & M & DI & 33 & 2007 & - & - & Aux. de Produção & Pepsico \\
\hline 21 & $\mathrm{M}$ & DI & 37 & 2013 & - & - & Aux. de Caixa & Sup. Nova Estrela \\
\hline 22 & M & DI & 48 & 2011 & - & - & Aux. de Produção & Klin \\
\hline 23 & M & DI & 29 & 2006 & 2015 & Saúde & Repositor & Posto Parati \\
\hline 24 & $M$ & DI & 42 & 2011 & - & - & Aux. de Produção & Klin \\
\hline 25 & $\mathrm{M}$ & DI & 26 & 2015 & - & - & Aux. de Produção & Pepsico Mabel \\
\hline 26 & M & DI & 30 & 2015 & 2015 & Comportamento & Aux. de Produção & Kid \\
\hline 27 & $\mathrm{~F}$ & DI & 42 & 2013 & - & - & $\begin{array}{l}\text { Auxiliar de } \\
\text { Recepção }\end{array}$ & Clinica CER II \\
\hline 28 & $\mathrm{M}$ & DI & 20 & 2013 & - & - & Aux. de Produção & $\begin{array}{l}\text { Alimentos Sta. } \\
\text { Cruz }\end{array}$ \\
\hline 29 & M & DI & 26 & 2015 & - & - & Aux. de Limpeza & Cargill \\
\hline
\end{tabular}

Em 2016, a crise econômica permanece em nosso país, e por esse motivo houve cortes na mão de obra em inúmeras empresas, ocasionando também uma queda, mesmo que pequena, mas ainda sim significativa para os deficientes intelectuais inseridos, mudando nosso quadro atual de 23 para 21 . Tabela 6. 
Tabela 6. DIs Inseridos e Acompanhados pela APAE de Três Lagoas/MS, em 2016.

\begin{tabular}{|c|c|c|c|c|c|c|c|c|}
\hline $\mathbf{N}^{\mathbf{o}}$ & Sexo & Def. & Idade & $\begin{array}{c}\text { Admis- } \\
\text { são }\end{array}$ & Desligado & $\begin{array}{l}\text { Motivo da } \\
\text { Saída }\end{array}$ & Função & Empresa \\
\hline 01 & M & DI & 40 & 2015 & - & - & Aux. de Produção & Adar \\
\hline 02 & M & DI & 25 & 2015 & 2016 & Saúde & Aux. de Produção & Emplal \\
\hline $\mathbf{0 3}$ & $\mathrm{M}$ & DI & 35 & 2014 & 2016 & Comportamento & $\begin{array}{l}\text { Aux. de Serviços } \\
\text { Gerais }\end{array}$ & Avanti \\
\hline 04 & $\mathrm{M}$ & DI & 33 & 2007 & - & - & $\begin{array}{l}\text { Supervisor de } \\
\text { Repositor }\end{array}$ & Pepsico Mabel \\
\hline 05 & $F$ & DI & 30 & 2009 & - & - & $\begin{array}{l}\text { Aux. de Serviços } \\
\text { Gerais }\end{array}$ & Cargill \\
\hline 06 & $F$ & DI & 41 & 2013 & - & - & $\begin{array}{l}\text { Repositora/Aux. de } \\
\text { Caixa }\end{array}$ & Pepsico Mabel \\
\hline 07 & $\mathrm{~F}$ & DI & 30 & 2013 & - & - & $\begin{array}{l}\text { Atendente de Serv. } \\
\text { Gerais }\end{array}$ & Enterhome \\
\hline 08 & $\mathrm{~F}$ & DI & 29 & 2015 & 2016 & Comportamento & Aux. de Limpeza & Cargill \\
\hline 09 & $\mathrm{~F}$ & DI & 22 & 2012 & 2016 & Comportamento & Aux. de Copeira & Cargill \\
\hline 10 & M & DI & 31 & 2013 & - & - & Aux. de Produção & Klin \\
\hline 11 & $\mathrm{~F}$ & DI & 23 & 2015 & - & & Aux. de Produção & Emplal \\
\hline 12 & M & DI & 42 & 2009 & - & - & $\begin{array}{l}\text { Aux. de } \\
\text { Almoxarifado }\end{array}$ & Cargill \\
\hline 13 & $\mathrm{~F}$ & DI & 25 & 2012 & 2016 & Saúde & Aux. de Produção & Klin \\
\hline 14 & M & DI & 25 & 2014 & - & - & Aux. de Limpeza & Cargill \\
\hline 15 & M & DI & 42 & 2005 & - & - & Empacotador & Sup. Nova Estrela \\
\hline 16 & M & DI & 27 & 2007 & - & - & $\begin{array}{l}\text { Aux. de Serviços } \\
\text { Gerais }\end{array}$ & Avanti \\
\hline 17 & M & DI & 33 & 2007 & - & - & Aux. de Produção & Pepsico \\
\hline 18 & $\mathrm{M}$ & DI & 37 & 2013 & - & - & Aux. de Caixa & Sup. Nova Estrela \\
\hline 19 & M & DI & 48 & 2011 & - & - & Aux. de Produção & Klin \\
\hline 20 & M & DI & 42 & 2011 & - & - & Aux. de Produção & Klin \\
\hline 21 & $\mathrm{M}$ & DI & 26 & 2015 & - & - & Aux. de Produção & Pepsico Mabel \\
\hline 22 & $\mathrm{~F}$ & DI & 42 & 2013 & 2016 & Comportamento & $\begin{array}{l}\text { Auxiliar de } \\
\text { Recepção }\end{array}$ & Clinica CER II \\
\hline 23 & M & DI & 20 & 2013 & - & - & Aux. de Produção & $\begin{array}{l}\text { Alimentos Sta. } \\
\text { Cruz }\end{array}$ \\
\hline 24 & $\mathrm{M}$ & DI & 26 & 2015 & - & - & Aux. de Limpeza & Cargill \\
\hline 25 & $\mathrm{M}$ & DI & 31 & 2007 & 2016 & Saúde & $\begin{array}{l}\text { Aux. de Serviço } \\
\text { Gerais }\end{array}$ & Transp. São Luiz \\
\hline 26 & $\mathrm{M}$ & DI & 19 & 2016 & - & - & Aux. de Produção & Pepsico \\
\hline 27 & M & DI & 19 & 2016 & - & - & Aux. de Produção & Pepsico \\
\hline 28 & M & DI & 19 & 2016 & - & - & Aux. de Produção & Pepsico \\
\hline
\end{tabular}

Desde 2011 a 2016, a APAE Três Lagoas inseriu cerca de 30 deficientes intelectuais no mercado de local, sendo que apenas 3 em supermercados, 1 em uma clinica, 1 em um posto de combustível e os demais em industrias, sobre tudo têxteis, de alimentos e de calçados infantis. O tempo médio de treinamento dos DIs, variaram nesse período, de 6 meses a 24 meses

A maior inclusão ocorreu de 2012 para 2013, com a inclusão de 8 DIs, graças a parceria com as empresas, que passam a cobrar acompanhamento integral por parte de APAE, através de visitas regulares ou quando a empresa tem algum problema com um inserido. 


\section{Apøe Ciênci@ Artigo}

A parceria deu muito certo, que praticamente todas as indústrias solicitam apoio da APAE, não apenas para os inseridos por ela, quanto também, para outras pessoas deficientes contratadas pelas indústrias, mostrando a confiança e o respeito alcançado pela instituição.

As entidades que se propõem treinamento profissional para inclusão de pessoas com deficiência, precisam, antes de nada, conhecer de forma clara a distinção entre profisssionalizar e preparar para o trabalho. Profissionalizar significa estar apto para exercer uma profissão, enquanto preparar para o trabalho é deixar apto para a vida produtiva por meio da prática que desenvolva a capacidade laborativa.

\section{CONSIDERAÇÕES FINAIS}

Nos dias atuais, encontramos uma evolução quantitativa bastante significativa, na inserção desses DIs nas empresas parceiras da APAE/TL, visto o resultado favorável que estes apresentam na execução do trabalho, sendo mais produtivos que as pessoas sem deficiências alguma, pois trabalham com amor. Como também, o suporte prestado pela APAE na inclusão dessas pessoas, proporcionando inserção e acompanhamento até a adaptação plena desses indivíduos, consequentemente, dissemina-se a conscientização entre os empresários, diminuindo as empresas que preferem pagar multas, em vez de contratar pessoas com deficiência.

A APAE Três Lagoas através da sua equipe de mercado de trabalho, integra a visão de que o DI a ser incluído, não tem apenas que passar por um treinamento de atividades profissionais e sim ser preparado para a vida e para ser feliz. Pois se notou que quando se encontram insatisfeitos em seus postos de trabalho ou com a empresa, estes solicitam que gostariam de ser transferidos para outra empresa. Não, em sua grande maioria devido a salários e sim porque querem ambiente de trabalho mais harmônico e colaborativo. Quando bem preparados e aceitos pela empresa e seus colegas de trabalho, trabalham felizes e com boa produtividade.

Como bem coloca Beserra e Domingues (2009) inclusão profissional de pessoas com DI, além de humanizar o ambiente de trabalho, promove a melhoraria o clima organizacional e acaba por influenciar positivamente a sociedade, ao destacar a importância de conviver e valorizar a diversidade, promovendo a igualdade de oportunidades para todos. 


\section{Apøe Ciênciø Artigo}

\section{REFERÊNCIAS}

APAE Três Lagoas Associação de Pais e Amigos dos Excepcionais - APAE de Três Lagoas. Relatórios Anuais do Mercado de Trabalho, anos de 2011 a 2015. Três Lagoas/MS, 2016.

BESERRA. E.G.; DOMINGUES, S.K. A Contribuição das Relações Públicas na Inclusão da Pessoa com Deficiência Mental no Mercado de Trabalho. Disponível em: Acesso em: 10/09/2009.

BRASIL Ministério do Trabalho. Relatorio Anual de informações Sociais 2014. $\quad$ http://www.brasil.gov.br/cidadania-e-justica/2015/09/numero-detrabalhadores-com deficiencia-cresce-no-mercado.

Lei n. 8.213, de 24 de Julho de 1991. Dispõe sobre os planos de benefícios da previdência social e dá outras providências. Disponível em:

$<$ http://www.planalto.gov.br/civil_03/leis/18213cons.htm> Acesso em: 21 junho. 2016.

Constituição da República Federativa do Brasil. Brasília: Senado, 1988.

Disponívelem:<http://www.planalto.gov.br/ccivil_03/constituicao/constitui-ção compilado.htm>. Acesso em: 10 junho. 2016.

FIRJAN Federação das Indústrias do Estado do Rio de Janeiro. Índice de Desenvolvimento Municipal (IFDM) 2013. Pesquisa e Estudos Socioeconômicos. Rio de Janeiro, 2015: 28p.

MACHADO, Gisele. C. Os caminhos da inclusão no mercado de trabalho. UNIJUÍ Universidade regional do noroeste do estado do Rio Grande do Sul. Ijuí/RS, 2012. (Especialista em Educação Especial - Deficiência Mental e Transtornos e Dificuldades de Aprendizagem).

MATO GROSSO DO SUL Diagnostico socioeconômico do Mato Grosso do Sul. Secretaria de Estado de Meio Ambiente e Desenvolvimento Econômico - SEMADE. Campo Grande, 2015: 134p. 